\title{
Computed Tomography of Paranasal Sinus Pathologies with Functional Endoscopic Sinus Surgery/Nasal Endoscopy Correlation
}

\author{
Kiran Rao
}

\section{ABSTRACT}

A prospective study conducted on 50 patients in Sri Guru Ram Das (SGRD) Institute of Medical Sciences and Research, Amritsar, India, suffering from paranasal sinus diseases correlating the findings of computed tomography (CT) using Siemens somatom emotion 6 slice CT machine with diagnostic nasal endoscopy or functional endoscopic sinus surgery (FESS).

Infection of the paranasal sinuses is very common. Surgical clearance of these chronically infected sinuses while maintaining their ventilation and drainage is the treatment of choice. To achieve this goal, there should be some diagnostic modality which guides us towards exact diagnosis and safe intervention. Computed tomography proves to be the most reliable method of preoperative assessment of patients undergoing FESS as it delineates the extent of the disease, define any anatomical variants and relationship of the sinuses with the surrounding important structures. Thus, providing a road map for sinus surgery.

In our study, most patients were in the 3rd and 4th decades of their life with equal disease incidence in males and females. The most common sinus involved was anterior ethmoid sinus while sphenoid sinus was least commonly involved. Commonest pattern of inflammation was sinonasal polyposis followed by osteomeatal unit pattern. On correlating CT diagnosis with final diagnosis, chronic sinusitis has $86 \%$ sensitivity and $96.5 \%$ specificity. Polyps have sensitivity of $96.15 \%$ and specificity of $95.83 \%$. Again for fungal sinusitis CT has lower sensitivity of $71.4 \%$ and specificity of $93.02 \%$. For diagnosing benign and malignant lesions CT has $100 \%$ sensitivity, specificity, could be due to small number of masses evaluated. This study proved that CT is the modality of choice for evaluation and planning the management of symptomatic patients of paranasal sinus pathologies.

Keywords: Computed tomography scan, Functional endoscopic sinus surgery, Paranasal sinus pathology.

How to cite this article: Rao K. Computed Tomography of Paranasal Sinus Pathologies with Functional Endoscopic Sinus Surgery/Nasal Endoscopy Correlation. Clin Rhinol An Int J 2015;8(1):15-19.

Associate Professor

Department of ENT, Sri Guru Ram Das Institute of Medical Sciences and Research, Amritsar, Punjab, India

Corresponding Author: Kiran Rao, Associate Professor Department of ENT, Sri Guru Ram Das Institute of Medical Sciences and Research, Amritsar, Punjab, India, Phone: 01832870200, e-mail: drkiranrao25@yahoo.com

\section{Source of support: Nil}

Conflict of interest: None

\section{INTRODUCTION}

Pathological lesions of the paranasal sinuses include a wide spectrum of conditions ranging from inflammation to neoplasms both benign and malignant. These sinuses are in close anatomical relationship with orbit, cranial fossa and pterygopalatine fossa. Hence, early involvement of these areas is an important feature. Since clinical assessment is hampered by the surrounding bony structures, diagnostic radiology is of paramount importance. ${ }^{1}$

While conventional plain radiography readily demonstrates maxillary and frontal sinus disease they provide limited views of the anterior ethmoid cells, the upper two thirds of the nasal cavity and the frontal recess. ${ }^{2}$

Computed tomography (CT) imaging provides detailed information of the paranasal sinuses and is now well established as an alternative to standard radiographs. ${ }^{3,4}$ Computed tomography scan has become modality of choice for evaluation of peripheral nervous system (PNS) pathologies as it optimally displays bony details, air and outlines soft tissue as well.

Infection of the paranasal sinuses is one of the commonest cause of patients visit to the otorhinolaryngologist. Surgical clearance of these chronically infected sinuses while maintaining their ventilation and drainage is the treatment of choice. To achieve this goal, there should be some diagnostic modality which guides us towards exact diagnosis and safe intervention. Over past decade, both CT and nasal endoscopy have been used successfully as diagnostic modality in sinus disease.

The principle of the surgery should be oriented towards opening up any restriction which might impair natural mucociliary clearance. To perform functional endoscopic sinus surgery effectively and safely, the surgeon must have detailed knowledge of the anatomy of the lateral nasal wall, paranasal sinuses and surrounding vital structures.

Computed tomography proves to be the most reliable method of preoperative assessment of patients undergoing functional endoscopic sinus surgery (FESS) as it delineates the extent of the disease, define any 
anatomical variants and relationship of the sinuses with the surrounding important structures - thus providing a road map for sinus surgery. As a rule, surgeons individualize their surgical approach according to the amount and location of disease they see on CT scan.

Endoscopic techniques for paranasal sinus surgery have allowed detailed and complete visualization of sinus disease while promising minimum distress to the patient. The telescopic view shows detail of the sinus anatomy and its disease. It has been possible to see areas of the cribriform plate and orbital wall that are at risk to produce cerebrospinal fluid rhinorrhea and orbital complications during the surgery.

Combination of diagnostic endoscopy and systematic understanding of the lateral nasal wall with CT in the coronal plane has become the corner stone in the evaluation of the PNS disease. This is the basis of the new concept of FESS.

\section{AIMS AND OBJECTIVES}

- To diagnose the various pathologies of paranasal sinuses, like inflammatory disease, neoplasms and other miscellaneous conditions.

- To correlate the findings of CT with those of FESS/ diagnostic endoscopy.

\section{MATERIALS AND METHODS}

A prospective study correlating findings of $\mathrm{CT}$ in paranasal sinus diseases with diagnostic endoscopy or FESS was conducted on 50 patients in the Department of Radiodiagnosis, Sri Guru Ram Das Institute of Medical Sciences and Research (SGRDIMSR), Amritsar in conjunction with Department of ENT, SGRDIMSR.

Patients attended the ENT outpatient department with symptoms, like postnasal discharge, nasal obstruction, heaviness in the head or dull ache over sinus, anosmia, constitutional symptoms, like malaise, mental apathy, sore throat, cough, hoarseness, eustachian tube dysfunction, etc. and who were willing to undergo diagnostic nasal endoscopy/FESS.

Slices of $1.25 \mathrm{~mm}$ were taken in axial planes. Coronal sections were reconstructed. Osteomeatal complex was best displayed by coronal plane. The anatomy in coronal plane was depicted in a way similar to what surgeon saw as the nasal vault was approached with an endoscope. Contrast agent omnipaque was used if indicated, at a calculated dose of $300 \mathrm{mg} / \mathrm{kg}$ weight as a single intravenous bolus injection after serum creatinine level was estimated.

As per Glicklich et al graded the severity of sinus disease on CT-PNS findings. It is classified as:

- Grade 0: Less than $2 \mathrm{~mm}$ mucosal thickening on any sinus wall.
- Grade 1: All unilateral disease or anatomic abnormalities.

- Grade 2: Bilateral disease limited to the ethmoid or maxillary.

- Grade 3: Bilateral disease with involvement of at least one sphenoid or frontal sinus.

- Grade 4: Pansinus disease.

In this study, CT findings were entered in the patient proforma. Lund-Mackay scoring as shown in Table 1, was done for comparison in case of inflammatory lesions.

$\begin{array}{ll}0 \text { Point } & \text { No abnormality } \\ 1 \text { Point } & \text { Partial opacification } \\ 2 \text { Points } & \text { Total opacification }\end{array}$

Total score recorded to show the severity of inflammatory disease.

Tasks force on rhino sinusitis sanctioned by the American Academy of Otolaryngology used Lund-Mackay CT staging system (Table 1) by scoring method. ${ }^{4}$ This same staging system was used in our study as reference.

Endoscopic sinus surgery tailored according to the CT scan, was carried out. Any polypoidal or mass lesions were debrided or biopsy taken for histopathological examination and fungal culture in selected cases. Computed tomography PNS findings were correlated with diagnostic endoscopic/FESS findings. Sensitivity and specificity of CT findings were calculated using FESS findings as standard with reference to mucosal thickening, polypoidal mass lesions, involvement of adjacent bones and soft tissue.

\section{OBSERVATIONS}

A prospective correlational descriptive study of 50 patients who underwent CT-PNS was done and correlated with the final diagnosis after diagnostic endoscopy/FESS.

Table 1: Lund-Mackay staging system for chronic rhinosinusitis

\begin{tabular}{lllll}
\hline \multirow{2}{*}{ Sinus } & & No & Partial & Total \\
Ant. ethmoid & R & 0 & 1 & 2 \\
& opacification & opacification \\
\hline Post ethmoid & R & 0 & 1 & 2 \\
& L & 0 & 1 & 2 \\
Maxillary & R & 0 & 1 & 2 \\
Frontal & L & 0 & 1 & 2 \\
& R & 0 & 1 & 2 \\
Sphenoid & L & 0 & 1 & 2 \\
& R & 0 & 1 & 2 \\
& L & 0 & 1 & 2 \\
Osteomeatal & R & 0 & 1 & 2 \\
Complex & L & 0 & Obstructed & \\
\hline
\end{tabular}


In this study of 50 patients, (Graph 1) 8 patients were less than 20 years of age. Eleven patients were between 21 and 30 years. Eleven patients were between 41 and 50 years of age. Highest number of patients were in the range of 31 and 40 years. Youngest patient was 8 years and eldest patient was 85 years old.

In present study, 24 (48\%) of total patients were females and $26(52 \%)$ of patients were males. Most of the patients had nasal obstruction $(60 \%)$ followed in decreasing order by headache $(56 \%)$, nasal discharge $(52 \%)$. The least common complaint was swelling in facial region.

Table 2 is showing various pathologies of paranasal sinuses on CT scan. Domain name system was seen in 22 (44\%) patients with equal number of cases showing deviation on either side. Concha bullosa was noted in $9(18 \%)$ patients with unilateral left sided predominance. Osteomeatal unit obstruction was observed in $42(84 \%)$ patients with bilateral involvement seen in $21(42 \%)$ patients.

Most commonly diseased sinus was anterior ethmoid sinus, followed in decreasing order were maxillary, posterior ethmoid, frontal and sphenoid sinuses.

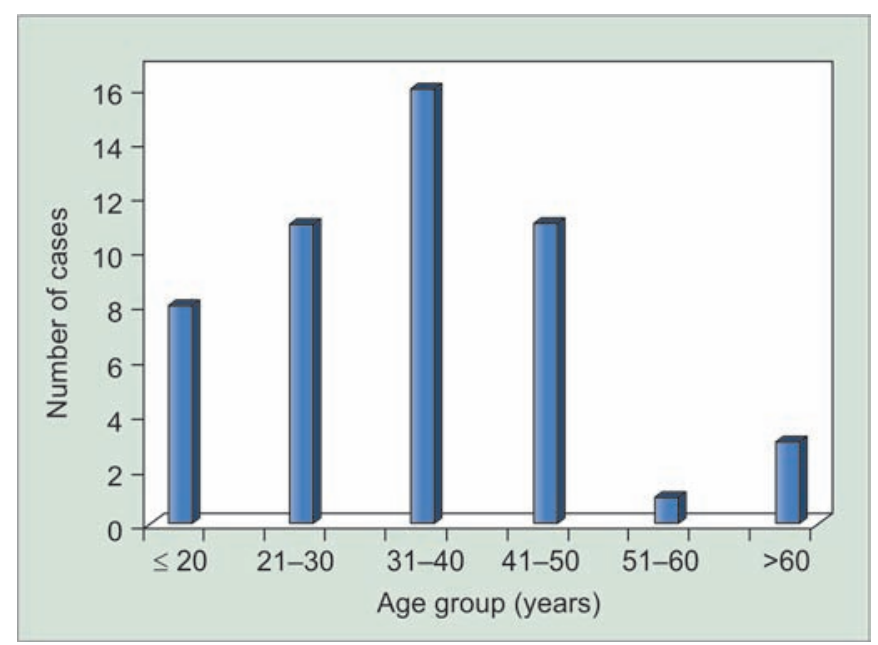

Graph 1: Age distribution of patients studied

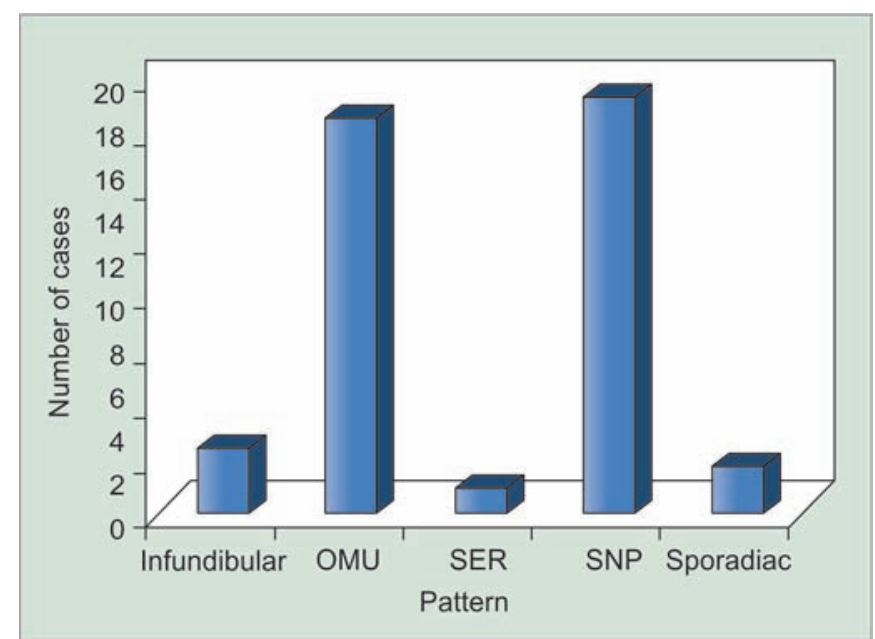

Graph 3: Patterns of inflammatory disease on CT
Graph 2 is showing severity of inflammatory disease and grading is done as per Glicklich et al study. Computed tomography severity was assessed in 43 patients who had inflammatory diseases. Maximum number 16 (37.2\%) of patients had grade 1 severity while grade 0 severity was found in none of the patients, least common was grade 2 seen in $6(14 \%)$ of patients.

Graph 3 showing patterns of inflammatory disease on CT. Sinonasal polyposis was the most common type of pattern involved (40\%), followed by OMU, infundibular, sporadic and sphenoethmoid recess patterns in decreasing order of involvement. Least common was sphenoethmoid type seen in $2 \%$ of cases.

As shown in Graph 4, endoscopic/FESS findings were similar to CT findings in $44(88 \%)$ patients and different from CT findings in $6(12 \%)$ patients. These different findings were related to either fungal disease or inspissated secretions.

Table 3 is showing Lund-Mackay scores of inflammatory diseases maximum number 14 (32.6\%) of patients had Lund-Mackay scores between 11 and 15 and minimum

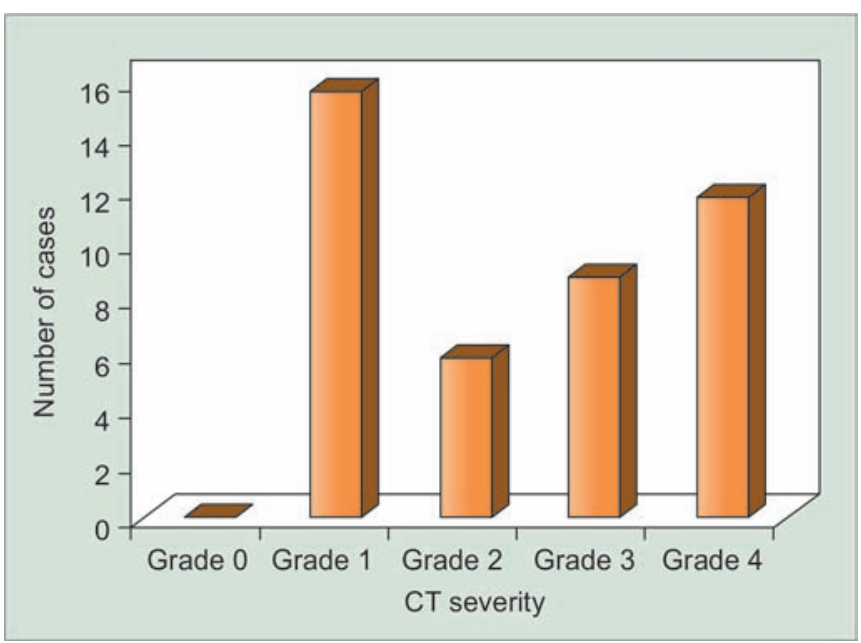

Graph 2: Computed tomography severity grading of inflammatory diseases

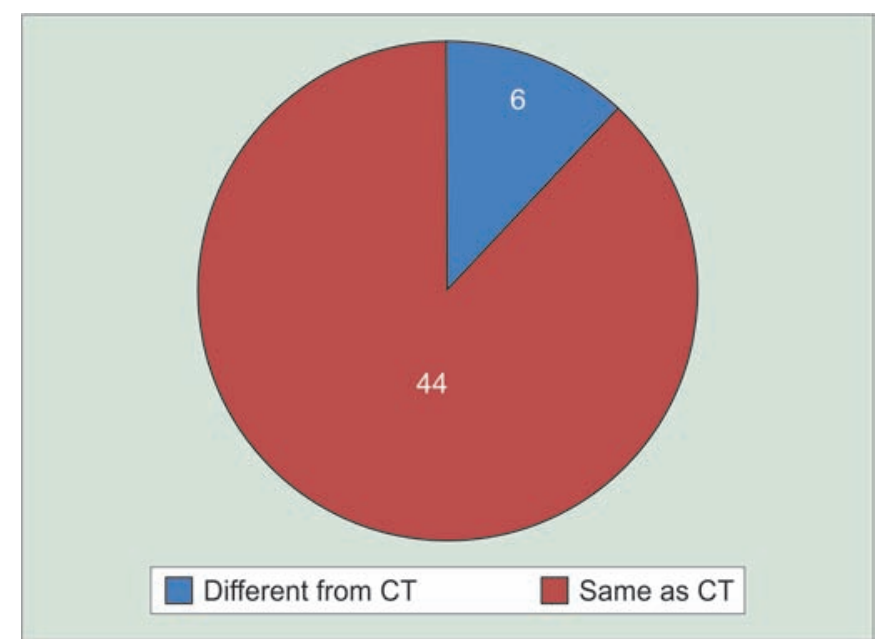

Graph 4: Endoscopy/FESS findings 
Table 2: Distribution of lesions according to CT-PNS

\begin{tabular}{lll}
\hline Investigations & Number $(n=50)$ & Percentage \\
\hline DNS & 28 & 56 \\
No & 11 & 22 \\
Left & 11 & 22 \\
Right & 22 & 44 \\
Total & & \\
Concha bullosa & 41 & 82 \\
No & 5 & 10 \\
Left & 1 & 2 \\
Right & 3 & 6 \\
Bilateral & 9 & 18 \\
Total & & \\
OMU obstruction & 8 & 16 \\
No & 15 & 30 \\
Left & 6 & 12 \\
Right & 21 & 42 \\
Bilateral & 42 & 84 \\
Total & &
\end{tabular}

Table 3: Lund-Mackay score showing severity of inflammatory diseases PNS

\begin{tabular}{lll}
\hline Lund-Mackay & Number $(n=43)$ & Percentage \\
\hline Score & & \\
$\leq 5$ & 7 & 16.3 \\
$6-10$ & 10 & 23.3 \\
$11-15$ & 14 & 32.6 \\
$16-20$ & 6 & 14 \\
$21-24$ & 6 & 14 \\
Total & 43 & 100 \\
Mean \pm SD & $12.09 \pm 6.20$ & \\
\hline
\end{tabular}

Table 4: Correlation of CT with final diagnosis

\begin{tabular}{llllll}
\hline Parameters & SEN & SP & PPV & NPV & Accuracy \\
\hline $\begin{array}{l}\text { Chronic } \\
\text { sinusitis }\end{array}$ & 86 & 96.5 & 94.7 & 90.32 & 92 \\
Polyps & 96.15 & 95.83 & 96.15 & 95.83 & 96 \\
Fungal sinusitis & 71.4 & 93.02 & 62.5 & 95.23 & 90 \\
Others & 100 & 100 & 100 & 100 & 100 \\
\hline
\end{tabular}

number $6(14 \%)$ each of patients had scores between 16 to 20 and 21 to 24 .

On correlating CT diagnosis with final diagnosis as shown in Table 4 chronic sinusitis has $86 \%$ sensitivity and $96.5 \%$ specificity. Polyps have sensitivity of $96.15 \%$ and specificity of $95.83 \%$. Again for fungal sinusitis CT has lower sensitivity of $71.4 \%$ and specificity of $93.02 \%$.

\section{DISCUSSION}

It is now generally accepted that $\mathrm{CT}$ is the optimum imaging method of demonstrating simple inflammatory disease to neoplasms in the paranasal sinuses. Clinical assessment can be used to evaluate acute sinus infection and $\mathrm{CT}$ is used for the investigation of persistent and chronic sinus disease refractory to medical therapy. Computed tomography evaluates the osteomeatal complex anatomy which is not possible with plain radiographs. Removal of disease in osteomeatal complex region is the basic principle of FESS which is best appreciated on CT scan.

This study was carried out to evaluate the pathological lesions of the paranasal sinuses by CT. Fifty patients were evaluated with $\mathrm{CT}$ who were referred after clinical assessment and, then correlated with diagnostic endoscopic/FESS.

In present study, the patients age ranged between 8 and 85 years which was consistent with study done by Prabhakar et al Maximum number of patients were aged between 31 and 40 years. ${ }^{5-7}$ In present study, $48 \%$ patients were females and $52 \%$ patients were males which was consistent with the study by Kirtane et al. ${ }^{8}$

Domain name system was seen in 22 of 50 patients constituting $44 \%$. Right DNS was seen in equal number of patients as left DNS. Concha bullosa was seen in $9(18 \%)$ patients and in literature it varied between 16 and 53\%.9-11 Osteomeatal units were involved in $42(84 \%)$ patients and bilateral involvement was seen in more number of patients than unilateral involvement. Hence, the overall incidence of inflammatory disease in the osteomeatal complex in symptomatic patients was no different with and without concha bullosa. Similar findings were observed in study by Maru and Gupta Y. ${ }^{11}$

Most common sinus involved was anterior ethmoid sinus in $38(76 \%)$ pts, followed by maxillary, posterior ethmoid, frontal and sphenoid sinuses in decreasing order. Studies in literature observed involvement of anterior ethmoid sinus and maxillary sinus more commonly. ${ }^{7}$ Present study correlates well with former study, where the number of patients studied were 60 and all patients underwent FESS. In all the studies sphenoid was least involved, which is also observed in this study $(48 \%)$. Sinonasal polyposis was the most common type of pattern involved, followed by osteomeatal unit, infundibular, sporadic and sphenoethmoid recess patterns in decreasing order of involvement.

The CT severity assessed for 43 patients with inflammatory diseases showed highest number of patients with grade 1, i.e. $16(37.2 \%)$ patients and lowest with grade 0 , i.e. $0(0 \%)$ patient. Lund-Mackay score between 11 and 15 was observed in most number of patients 14 (32.6\%). The mean LMKS score observed was $12.09 \pm 6.20$.

Greatest pitfall in diagnosis of PNS diseases by $\mathrm{CT}$ is the fungal sinusitis. In this study, eight patients were studied among which five $(71.4 \%)$ were diagnosed correctly and others were not diagnosed on CT. The sensitivity was $71.4 \%$ and specificity was $93.02 \%$ for CT to diagnose fungal sinusitis. The sensitivity described in literature was $76 \%$ by Zenreich et al which was a retrospective study. 
Endoscopic findings were almost all correlated with CT findings except in Fungal sinusitis. The findings of CT were similar to diagnostic endoscopy/FESS findings in $44(88 \%)$ of patients and different in $6(12 \%)$ patients. All the false positives or false negatives were related to fungal sinusitis. Except the fungal sinusitis, sensitivity and specificity of CT was high.

On correlating CT diagnosis with final diagnosis, chronic sinusitis has $86 \%$ sensitivity and $96.5 \%$ specificity. ${ }^{12,13}$ Polyps have sensitivity of $96.15 \%$ and specificity of $95.83 \%{ }^{13}$ Again for fungal sinusitis CT has lower sensitivity of $71.4 \%$ and specificity of $93.02 \% \cdot{ }^{14,15}$ For diagnosing benign and malignant lesions CT has 100\% sensitivity, specificity, positive predictive value and negative predictive value with $100 \%$ accuracy. ${ }^{16}$ This high sensitivity and specificity for benign and malignant masses could be due to small number of masses evaluated.

Thus, CT plays an important role in diagnosing and also adding important findings for the better management of the patients with paranasal sinus diseases. It is cost effective and less time consuming modality compared to MRI, CT is the modality of choice. ${ }^{17}$

\section{CONCLUSION}

This was the prospective correlational descriptive clinical study carried out on 50 symptomatic paranasal sinus diseased patients who underwent $\mathrm{CT}$ paranasal sinuses in both coronal and axial sections. Most patients were in the 3rd and 4th decades of their life with equal disease incidence in males and females. The most common complaint with which they presented was nasal obstruction followed by headache and nasal discharge. On evaluating patients with CT PNS, the most common sinus involved was anterior ethmoid sinus while sphenoid sinus was the least involved. Commonest pattern of inflammation was sinonasal polyposis followed by osteomeatal unit pattern. Sensitivity and specificity of $\mathrm{CT}$ in diagnosing fungal sinusitis was 71.4 and $93.02 \%$ respectively. But sensitivity and specificity for detection of mucosal abnormality was very good. Computed tomography had best statistical results in evaluating benign and aggressive lesions, which was $100 \%$ in this study attributable to the less number of aggressive or malignant lesions studied. On the other hand, clinical assessment of these lesions was poor, indicates that $\mathrm{CT}$ is mandatory in assessment of paranasal sinus diseases and also to look for any bone erosion or destruction with adjacent structure involvement.

Computed tomography findings correlated well and were same as those of diagnostic endoscopic/FESS findings in $44(88 \%)$ cases which was confirmed on histopathology report (HPR). In remaining $6(12 \%)$ cases, $\mathrm{CT}$ findings were different from those of diagnostic
endoscopy/FESS. All these cases were related to fungal sinusitis as confirmed on HPR later. To conclude, this study proved that $\mathrm{CT}$ is the modality of choice for evaluation and planning the management of symptomatic patients of paranasal sinus pathologies. Fungal sinusitis and dense secretions are potential pitfalls on CT to differentiate them. But, CT may suggest fungal sinusitis in whom it is not suspected. It is the modality of choice in evaluating the bone erosion or destruction. Computed tomography evaluation of PNS in symptomatic patients helps in planning the further management of the patient.

\section{REFERENCES}

1. Parsons C, Hodson N. Computed tomography of paranasal sinus tumours. Radiology 1979 Sep;132:641-645.

2. Zinreich SJ. Paranasal sinus imaging. Otolaryngol Head Neck Surg 1990;130:863.

3. White PS, Cowan IA, Robertson MS. Limited CT scanning techniques of the paranasal sinuses. J Laryngol Otology 1991 Jan;105:20-23.

4. Shroff MM, Shetty PG, Navani SB, Kirtane MV. Coronal screening sinus $\mathrm{CT}$ in inflammatory sino-nasal disease. IJRI 1996;6:3-17.

5. Venkatachalam VP, Bhat A. Functional endoscopic sinus surgery: a newer surgical concept in the management of chronic sinusitis. Ind J Otolaryngol Head Neck Surg 1999 Dec;52(1):13-22.

6. Gliklich RE, Metson R. Techniques for outcomes research in chronic sinusitis. Laryngoscope 1995 Apr;105:387-390.

7. Prabhakar S, Mehra YN, Talwar P, Mann SBS, Mehta SK. Fungal infections in maxillary sinusitis. Ind J Otolaryngol Head Neck 1992 Jun;1(2):54-58.

8. Kirtane MV. Functional endoscopic sinus surgery: a preliminary study. Ind J Otolaryngol 1991;43:126-129.

9. Asruddin, Yadav SPS, Yadav RK, Singh J. Low dose CT in chronic sinusitis. Ind J Otolaryngol Head Neck Surg 2000 Mar;52(1):17-22.

10. Lloyd GAS, Lund VJ, Scadding GK. CT of the paranasal sinuses and functional endoscopic surgery: a critical analysis of 100 symptomatic patients. J Laryngol Otology 1991 Mar; 105:181-185.

11. Maru YK, Gupta Y. Concha bullosa: frequency and appearances on sinonasal CT. Ind J Otolaryngol Head Neck Surg 2000 Mar;52(1):40-44.

12. Bhattacharyya N, Jones DT. The diagnostic accuracy of computed tomography in chronic rhinosinusitis. Arch Otolaryngol Head Neck Surg 2004 Sep;130(9):1029-1032.

13. Younis RT, Anand VK, Davidson B. The role of computed tomography and magnetic resonance imaging in patients with sinusitis with complications.Laryngoscope 2002 Feb;112(2):224-229.

14. Zinreich SJ, Kennedy DV, Malat J. Fungal sinusitis: diagnosis with CT and MR imaging. Radiology 1988;169:439-444.

15. Dhong HJ, Jung JY, Park JH. Diagnostic accuracy in sinus fungus balls: CT scan and operative findings. Am J Rhinol 2000 Aug;14(4):227-230.

16. Matsubara K. Application of CT in diagnosing carcinoma of the maxillary sinuses. AJ Med 1982;44:57-67.

17. Aygun N, Zinreich SJ. Imaging for functional endoscopic sinus surgery. OCNA 2006;39:403-416. 